“C 2013 IEEE. Personal use of this material is permitted. Permission from IEEE must be obtained for all other uses, in any current or future media, including reprinting/republishing this material for advertising or promotional purposes, creating new collective works, for resale or redistribution to servers or lists, or reuse of any copyrighted component of this work in other works." 


\title{
Admittance Control Scheme for Implementing Model-based Assistance-As-Needed on a Robot
}

\author{
Marc G. Carmichael and Dikai Liu
}

\begin{abstract}
A model-based assistance-as-needed paradigm has been developed to govern the assistance provided by an assistive robot to its operator. This paradigm has advantages over existing methods of providing assistance-as-needed for applications such as robotic rehabilitation. However, implementation of the model-based paradigm requires a control scheme to be developed which controls the robot so as to provide the assistance calculated by the model-based paradigm to its operator.

In this paper an admittance control scheme for providing model-based assistance-as-needed is presented. It is developed considering its suitability for human-robot interaction, and its role within the model-based assistance-as-needed framework. Results from the control implemented on an example robot showed it is capable of providing the operator with the desired level of assistance as governed by the model-based paradigm. This is an essential requirement for the paradigm to be capable of providing efficacious assistance-as-needed in applications such as robotic rehabilitation.
\end{abstract}

\section{INTRODUCTION}

In robotic rehabilitation most paradigms are assistive, with the robot assisting the patient as they manoeuvrer their impaired limb as part of therapy [1]. There is much interest in developing new control methods to improve patient recovery. A promising paradigm is assistance-as-needed (AAN) where the objective is to provide the patient with the minimum robotic assistance they require to perform therapies. This has shown promising results in neuro-rehabilitation as AAN inherently promotes active participation of the patient which is essential for motor-neuron recovery [2]. A common approach towards implementing AAN is to use performance-based methods where the patient's assistance needs are inferred from their performance, and then used to adjust the robot's assistance accordingly [3]-[5].

A model-based paradigm for providing AAN has been developed using models to calculate the assistance needs of subjects for different upper limb tasks [6]-[8]. Their strength capability is calculated from a musculoskeletal model, which is then compared to the strength required to perform the tasks to gauge their assistance needs. This paradigm has several benefits compared to performance-based AAN methods. Since their assistance need is derived from a model, the operator is not required to perform tasks before it can be calculated, unlike performance-based methods which require tasks to be observed before their needs can be inferred from their performance. Secondly, incorporating a musculoskeletal model allows investigations to be performed relating to the

M. G. Carmichael and D. Liu are with the Centre for Autonomous Systems (CAS), Faculty of Engineering and IT (FEIT), University of Technology, Sydney (UTS), NSW 2007, Australia. Email: marc.carmichael@uts.edu.au, dikai.liu@uts.edu.au physiology of the operator. For example the musculoskeletal model can be used to identify impairments and to plan optimized therapies [9]-[11]. This is a significant advantage in applications such as rehabilitation which have an emphasis on the physiology of the operator.

To implement the model-based AAN paradigm onto a robotic system requires an appropriate control scheme to be developed. This control scheme operates the robot to provide its operator with assistance as calculated by the model-based paradigm. There are several considerations to be made, such as its suitability for human-robot interaction, and its ability to provide a controllable level of assistance to the operator. This paper develops an admittance control scheme to implement the model-based AAN paradigm on assistive robots. Section II details the model-based AAN paradigm and the role of the control scheme within its framework. Section III details the admittance control scheme and how it provides the operator with assistance based on their calculated needs. Section IV presents results of the control scheme implemented on an example robotic system which shows it is successful in providing the operator with assistance governed by the model-based AAN paradigm.

\section{MODEL-BASED AAN FRAMEWORK}

The framework for the model-based assistance-as-needed paradigm [8] is shown in Fig. 1. At the higher level of the framework two models are used to calculate the operator's assistance needs. A strength model calculates the strength capability of the operator at their hand in a specified direction in the task space. This strength is derived from an upper limb musculoskeletal model representing the operator. A second task model calculates the strength required by the task that is being performed. The task's strength requirement is compared with the operator's strength capability to

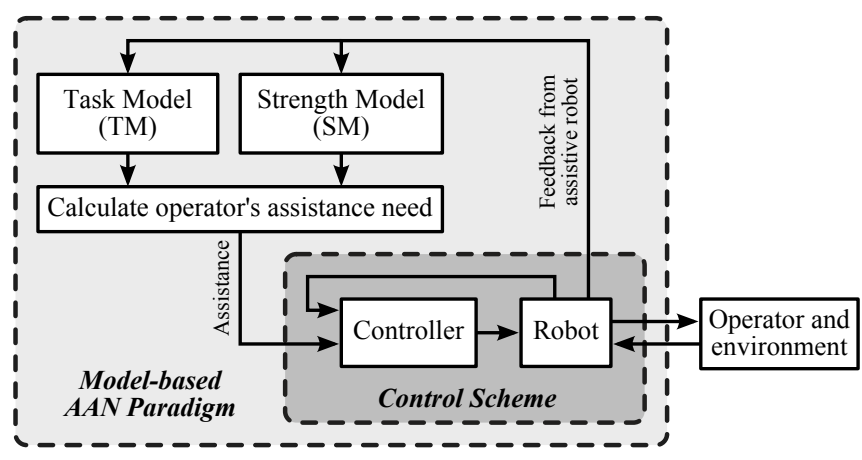

Fig. 1: Framework for the model-based AAN paradigm. 
gauge their assistance needs. Hence the assistance need is calculated considering the requirements of the task being performed, and since a musculoskeletal model is used, it also considers the modeled physiology of the operator. This allows physiological effects such as muscular impairments to be considered when calculating of the operator's assistance needs. Additional details regarding the calculation of operator strength and assistance need can be found in the following literature [6]-[8].

Fig. 2 shows an example of a subject's upper limb strength calculated at the hand [8]. The strength is visualized as a polar plot with the distance between a point on the plot and the origin (at the hand) proportional to the calculated strength, and the direction from the point to the polar origin corresponding to the direction in which the strength is calculated for. Two polar plots are shown, the first showing strength calculated with no physical impairment, and the second with impairment simulated in the biceps muscle. The arrows highlight two particular directions in the plots. It is seen that in one direction the strength is seen to reduce as a result of the impairment, whereas the second has no calculated decrease in strength. This demonstrates how the model-based AAN paradigm can estimate the assistance needs of the operator in different task space directions, as well as how muscular impairments affect their assistance needs and which directions are most affected.

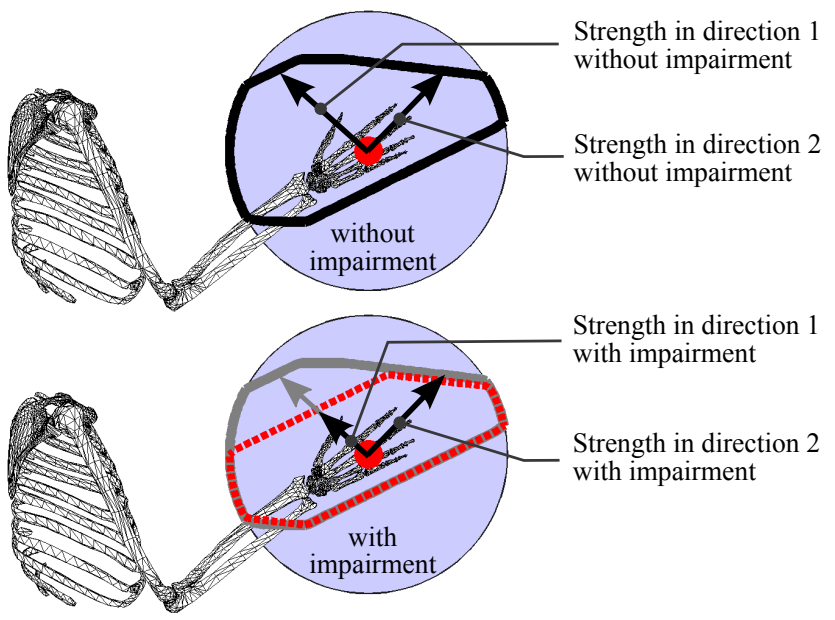

Fig. 2: Example of calculating assistance need using the model-based AAN paradigm. The two plots show the calculated strength at the hand with and without impairment in the biceps muscle. Arrows in each plot denote directions of interest. It's seen that impairment reduces strength in direction 1, whilst strength in direction 2 remains the same.

Results from this model-based calculation of the operator's assistance needs are fed to the lower level of the framework where they are used to govern the assistance provided by a robot. A variety of different control schemes may be used at the low level, as long as they are capable of providing the operator with the assistance calculated. This is an advantage as the choice of control scheme is often limited by the application or by the construction of the robot itself. In the next section an admittance control scheme is developed.

\section{Development of Control Scheme For the Model-BASEd AAN PARADIGM}

\section{A. Appropriate types of control schemes}

The control scheme is required to be suitable with respect to human-robot physical interaction. Motion control is a fundamental field in robotics, however by itself it is not suited to applications where robots interact physically with humans as it requires accurate task planning, and human collaboration is inherently difficult to define. Instead it is desirable to use force-based control schemes, since force control is essential for achieving robust behavior in poorly structured environments and for safe operation in the presence of humans [12]. Force control can be categorized into two types, direct or indirect force control [13]. Direct force control uses measurements from sensors to create a closed force feedback loop. An example is hybrid force control [14] where force and position in orthogonal directions at the robot's end effector are controlled simultaneously. This scheme requires a model of the environment which in most practical situations is not available [13]. Indirect schemes control force through the motion of the robot and are commonly used in applications such as robotic rehabilitation. A model of a dynamic system (typically spring-mass-damper) is used to relate robot interaction forces with the environment (including the human operator) to its motion. Schemes are commonly based on damping, stiffness, impedance, or admittance [15]-[18]. Such schemes control the robot to behave as the defined dynamic system in response to external interactions. For example impedance control measures the positional deviation of the robot (e.g. resulting from external interaction) and then controls its actuator forces such that the desired impedance is produced. Alternatively, admittance control measures interaction forces between the robot and environment using sensors, then generates an appropriate motion trajectory which it follows accordingly.

In this paper an admittance control scheme is chosen for development. This scheme may be used with various robotic platforms to implement the model-based AAN paradigm, however it is noted that the suitability of this and other forcecontrol schemes depends on the construction and actuation of the robotic system. Admittance control requires measurements of the interaction forces between the robot, operator, and the environment; as well as accurate trajectory following capabilities. Hence this implies that a robot with high power actuators and a stiff construction is preferred, which puts specific demands on the robot's design. Impedance control alternatively requires a robot with accurate position sensing, and is capable of precise force actuation. This implies that actuators should be almost ideal force sources and therefore be lightweight, low friction and low impedance [19].

\section{B. An admittance control scheme}

The model-based AAN paradigm is based around the strength at the hand of the operator [8]. As it is admittance based, force sensors are required where the robot interacts 


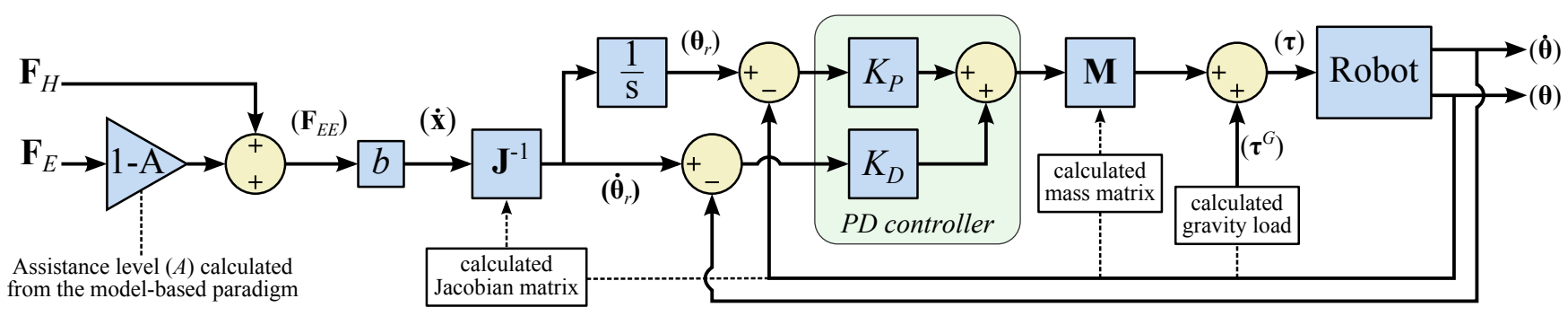

Fig. 3: The admittance control scheme developed to provide assistance to the operator as governed by the model-based AAN paradigm. Assistance is governed by the $A$ parameter set by the model-based AAN paradigm [6]-[8], ranging from 0 to 1 .

with the operator and the environment as tasks are performed. We assume that physical interaction with the robot only occurs at its end effector, with sensors available to measure the interaction. Fig. 3 shows the assistance control scheme developed to operate the robot such that a controllable amount of assistance is administered to the operator. Parameters $\mathbf{F}_{H}$ and $\mathbf{F}_{E}$ are vectors in task space coordinates representing the measured force interaction of the robot with both the operator and the task being performed, respectively. The forces are combined into a net force at the robot's end effector, represented as $\mathbf{F}_{E E}$, then multiplied by admittance gain $(b)$ to produce a reference end-effector velocity in task space coordinates $(\dot{\mathrm{x}})$. The kinematic Jacobian matrix (J) relating robot joint velocity to end-effector velocity is calculated for its current pose. Its inverse is used to transform the task space trajectory into an equivalent velocity trajectory in the robot's joint space (represented as $\dot{\boldsymbol{\theta}}_{r}$ ) and is integrated to create reference joint position trajectory $\boldsymbol{\theta}_{r}$.

The PD controller tracks the reference motion in joint space using a simple form of computed torque control [20]. Error between the measured joint position and velocity $(\boldsymbol{\theta}, \dot{\boldsymbol{\theta}})$ and the reference trajectories $\left(\boldsymbol{\theta}_{r}, \dot{\boldsymbol{\theta}}_{r}\right)$ are multiplied by gains $K_{P}$ and $K_{D}$ respectively. These were manually tuned with $K_{P}$ as high as could be achieved without causing instability as to increase disturbance rejection, and $K_{D}$ tuned to achieve the desired damping. Controller output is multiplied by the dynamic system's mass matrix $\mathbf{M}$, which is calculated continuously as a function of the robot's current position. The outcome is the desired joint torque $\tau$ to be actuated at

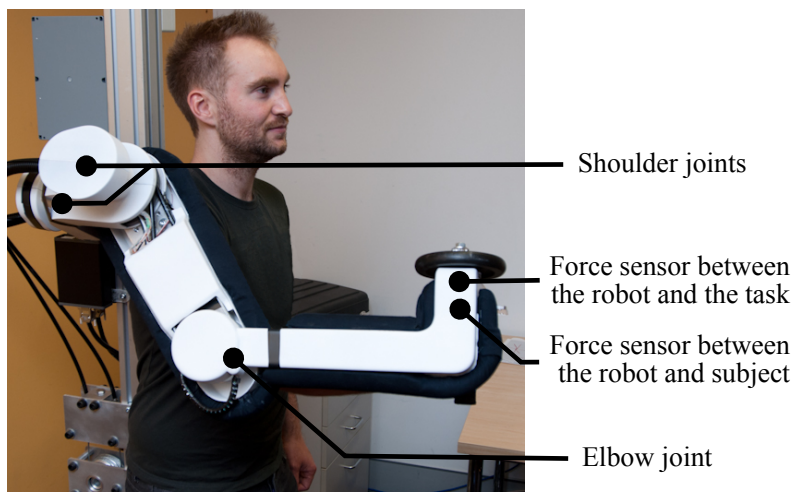

Fig. 4: The example robotic platform to validate the admittance control scheme. the joints of the robot. The gravity load at each joint due to the robot's own weight $\left(\tau^{G}\right)$ is calculated from a model of the robot and added to the torque output to linearize the system. The desired torque is then actuated depending on the hardware of the robot. For example if DC motors are used, the motor current required to achieve the desired torque may be calculated based on the motor's intrinsic torque constant, and then provided using current-controlled motor drivers. The result of this control scheme is a robot which is actively compliant, with its end-effector moving in response to the measured forces at its end effector.

\section{Controlling assistance}

To provide assistance to the operator the measured force of the task being performed $\left(\mathbf{F}_{E}\right)$ can be scaled down before it is combined with $\mathbf{F}_{H}$ and used to generate the robot's motion trajectory. We define parameter $A$ ranging from $0 \leq A \leq 1$ to represent the level of assistance the operator is provided. $A=$ 0 means zero assistance is provided, and $A=1$ means that the robot is providing all of the force required to perform the task. The force $\mathbf{F}_{E}$ is scaled by a gain of $1-A$, so when $A=$ 0 a gain of 1 is applied. In this scenario, to statically oppose the external force the operator must provide an opposite force at the end effector of equal magnitude such that $\mathbf{F}_{E E}=0$ and consequently no robot motion is generated. As assistance is increased (i.e. $A$ is increased) the force required from the operator to oppose the external task force becomes less and less, until $A=1$ when force $\mathbf{F}_{E}$ is scaled down to zero and no effort at all is required from the operator to oppose it. The model-based AAN paradigm sets the $A$ parameter based on the calculated assistance needs of the operator. This is a convenient method of providing assistance to the operator which can be dynamically controlled as tasks are performed.

\section{IMPLEMENTATION ON AN EXAMPLE ROBOT}

The admittance control scheme is implemented on an example assistive robot to validate its ability to provide the operator with a controllable level of assistance. The robot (shown in Fig. 4) is an exoskeleton designed to assist the upper limb of its operator using three active degrees of freedom (two at the shoulder, one at the elbow). The operator couples with the robot at their hand by holding a handle, in which a 6-axis load cell measures the interaction force corresponding to the term $\mathbf{F}_{H}$ in the control scheme. A number of experimental tasks were performed where a 
subject was required to oppose a load equivalent to $5 \mathrm{~kg}$ in various directions at the hand. The subject was defined as having impairments in varying muscle groups. The assistance provided by the robot is calculated for each task based on how their strength at the hand is affected by the muscle impairment. For example if the calculated strength decreased by $75 \%$, the assistance would be set $A=0.75$. The method used to calculate subject strength is detailed in [6].

The actual assistance the subject received at their hand is represented as $A_{H}$. This is calculated as the difference between the measured force the subject applied to the handle and the magnitude of the task load (i.e. $5 \mathrm{~kg}$ ), normalized by the task load. This is plotted against the desired assistance to be provided (i.e. $A$ ) in Fig. 5 for all of the experimental tasks that were performed. The results show a strong linear relationship between the desired assistance to provide the subject and the actual assistance they received at their hand, with a calculated correlation of $\rho=0.995$. This demonstrates that the robotic exoskeleton using the developed admittance control scheme was capable of providing the subject with the assistance calculated by the model-based paradigm.

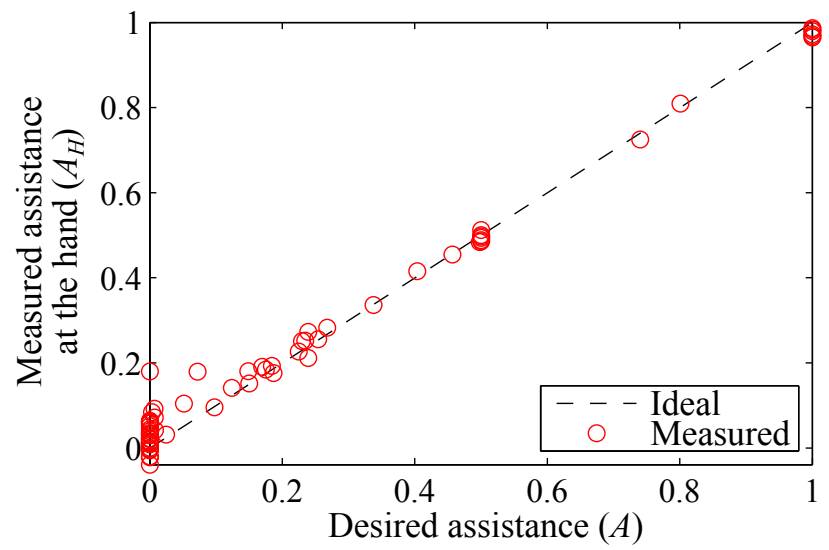

Fig. 5: The desired assistance $(A)$ calculated from the modelbased AAN paradigm, versus the actual assistance received at the hand $\left(A_{H}\right)$ measured using the robot's sensors.

\section{CONCLUSION}

An admittance control scheme to provide assistance as part of a model-based assistance-as-needed paradigm was developed. The control was developed considering the suitability for human-robot interaction and its integration into the model-based AAN framework. Results obtained using an experimental robot showed the control scheme is capable of providing a controllable level of assistance, which is an essential requirement for the model-based AAN paradigm. In future work we plan to utilize this control to provide modelbased AAN and analyze its efficacy to provide assistance suited to the needs of the operator.

\section{REFERENCES}

[1] L. Marchal-Crespo and D. J. Reinkensmeyer, "Review of control strategies for robotic movement training after neurologic injury,"
Journal of NeuroEngineering and Rehabilitation, vol. 6, no. 1, p. 20, 2009.

[2] N. Hogan, H. I. Krebs, B. Rohrer, J. J. Palazzolo, L. Dipietro, S. E. Fasoli, J. Stein, R. Hughes, W. R. Frontera, D. Lynch, and B. T. Volpe, "Motions or muscles? Some behavioral factors underlying robotic assistance of motor recovery." Journal of rehabilitation research and development, vol. 43, no. 5, pp. 605-618, 2006.

[3] H. Krebs, J. Palazzolo, L. Dipietro, M. Ferraro, J. Krol, K. Rannekleiv, B. Volpe, and N. Hogan, "Rehabilitation robotics: Performance-based progressive robot-assisted therapy," Autonomous Robots, vol. 15, pp. 7-20, 2003.

[4] J. Emken, J. Bobrow, and D. Reinkensmeyer, "Robotic movement training as an optimization problem: designing a controller that assists only as needed," in 9th International Conference on Rehabilitation Robotics (ICORR), 2005, June 2005, pp. 307 - 312.

[5] E. Wolbrecht, V. Chan, V. Le, S. Cramer, D. Reinkensmeyer, and J. Bobrow, "Real-time computer modeling of weakness following stroke optimizes robotic assistance for movement therapy," in Neural Engineering, 2007. CNE '07. 3rd International IEEE/EMBS Conference on, May 2007, pp. $152-158$.

[6] M. G. Carmichael and D. Liu, "Towards using musculoskeletal models for intelligent control of physically assistive robots," in Engineering in Medicine and Biology Society,EMBC, 2011 Annual International Conference of the IEEE, Sept 2011, pp. $8162-8165$.

[7] — "A task description model for robotic rehabilitation," in Engineering in Medicine and Biology Society,EMBC, 2012 Annual International Conference of the IEEE, 2012.

[8] _ - "Estimating physical assistance need using a musculoskeletal model," IEEE Transactions on Biomedical Engineering, 2013, (in press).

[9] J. Ueda, D. Ming, V. Krishnamoorthy, M. Shinohara, and T. Ogasawara, "Individual muscle control using an exoskeleton robot for muscle function testing," Neural Systems and Rehabilitation Engineering, IEEE Transactions on, vol. 18, no. 4, pp. 339 -350, Aug. 2010.

[10] B. J. Fregly, M. L. Boninger, and D. J. Reinkensmeyer, "Personalized neuromusculoskeletal modeling to improve treatment of mobility impairments: a perspective from european research sites." J Neuroeng Rehabil, vol. 9, p. 18, 2012.

[11] Y. Pei, Y. Kim, G. Obinata, E. Genda, and D. Stefanov, "Robot-aided rehabilitation task design for inner shoulder muscles," in Engineering in Medicine and Biology Society (EMBC), 2012 Annual International Conference of the IEEE, Sep 2012, pp. 3922 -3925.

[12] L. Villani and J. D. Schutter, "Force control," in Handbook of Robotics, B. Siciliano and O. Khatib, Eds. Berlin: Springer, 2008, ch. 7, pp. 161-185.

[13] B. Siciliano and L. Villani, Robot Force Control. Kluwer Academic, 1999.

[14] M. H. Raibert and J. J. Craig, "Hybrid position/force control of manipulators," Journal of Dynamic Systems, Measurement, and Control, vol. 103, no. 2, pp. 126-133, 1981.

[15] D. E. Whitney, "Force feedback control of manipulator fine motions," Journal of Dynamic Systems, Measurement, and Control, vol. 99, no. 2, pp. 91-97, 1977.

[16] J. K. Salisbury, "Active stiffness control of a manipulator in cartesian coordinates," in Decision and Control including the Symposium on Adaptive Processes, 1980 19th IEEE Conference on, vol. 19, Dec. 1980, pp. $95-100$.

[17] N. Hogan, "Impedance control: An approach to manipulation: Part I - theory; part II - implementation; part III - applications," Journal of Dynamic Systems, Measurement, and Control, vol. 107, no. 1, pp. $1-24,1985$.

[18] H. Kazerooni, T. Sheridan, and P. Houpt, "Robust compliant motion for manipulators, part I: The fundamental concepts of compliant motion," Robotics and Automation, IEEE Journal of, vol. 2, no. 2, pp. 83 - 92, Jun 1986.

[19] H. van der Kooij, J. Veneman, and R. Ekkelenkamp, "Compliant actuation of exoskeletons," in Mobile Robots: towards New Applications, A. Lazinica, Ed. InTech, 2006, ch. 7.

[20] W. Chung, L.-C. Fu, and S.-H. Hsu, "Motion control," in Handbook of Robotics, B. Siciliano and O. Khatib, Eds. Berlin: Springer, 2008, ch. 6, pp. 133-159. 